C-A/AP/\#152

May 2004

\title{
Quadrupole First RHIC Interaction Regions
}

S. Tepikian and S. Peggs

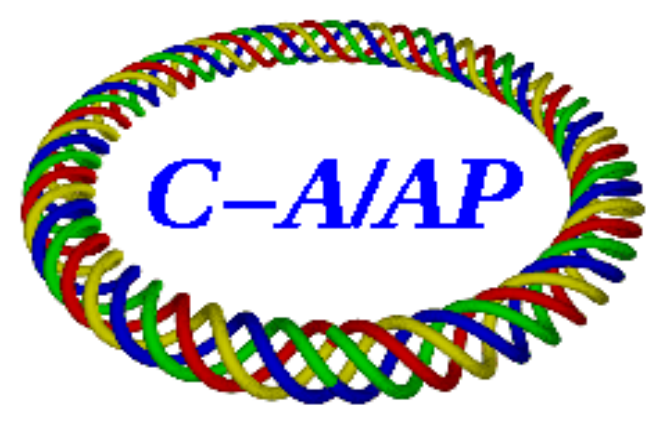

Collider-Accelerator Department Brookhaven National Laboratory Upton, NY 11973 
$\mathrm{CAD} / \mathrm{AP} / 152$

May 26, 2004

\title{
Quadrupole First RHIC Interaction Regions
}

\author{
S. Tepikian and S. Peggs
}

\section{Abstract}

How much luminosity can be gained by going to "quadrupole first" interaction region (IR) optics, in which the locations of the RHIC IR triplet and the beam separation dipoles are reversed, so that focusing occurs first, and beam separation second? Two scenarios are presented.

A luminosity enhancement factor of at least 5 is possible in the "outside 9.8" scenario, in which the inner face of the first quadrupole Q1 is placed at $s= \pm 9.8$ meters from the interaction point (IP), the current location of the inner face of the DX magnets. This scenario is trivially consistent with current RHIC detectors STAR and PHENIX.

A luminosity enhancement factor of at least 7 - perhaps as large as 10 - is possible in the "inside 9.8" scenario, when Q1 is permitted inside the $s= \pm 9.8$ meter boundary. This is consistent, for example, with the speculative design of a New Comprehensive Detector that is currently under discussion [1].

In both scenarios the IR quads are new magnets, upgraded to state-of-the-art performance parameters. The same old DX and D0 beam separation dipoles are reused in new locations.

The caveats and consequences of a quadrupole first IR are listed, but are barely addressed. The most important consequence is that proton-gold collisions are no longer possible. Further investigation is required before the viability of deuteron-gold collisions can be conclusively determined.

\section{Introduction}

The luminosity $L$ is

$$
L=F_{\text {coll }}\left(\frac{N^{2}}{4 \pi \sigma^{2}}\right)
$$

where $F_{\text {coll }}$ is the collision frequency, $N$ is the bunch population, and $\sigma$ is the transverse beam size at the collision point. It is assumed that the beam is round - both the beam sizes and the collision betas $\beta^{*}$ are the same in both planes. It is convenient to re-write this as

$$
L=M \xi^{2} \sigma^{\prime 2} \times\left(\frac{4 \pi \gamma^{2}}{r_{0}^{2}}\right) F_{r e v}
$$

where $\gamma$ is the Lorentz factor, $r_{0}$ is the classical radius of the appropriate ion, and $F_{r e v}$ is the revolution frequency. This shows that the goal of an optics upgrade is to increase $\sigma^{\prime}$ - the RMS angular beam size at the IP - as far as possible, under the assumption that the number of bunches in each beam $M$ and the beam-beam parameter $\xi$ are both held fixed for other reasons.

The upper limit to $\sigma^{\prime}$ is given by the need to limit the maximum beam size $\widehat{\sigma}$ in the triplet, since these two quantities increase together through the exact expression

$$
\widehat{\sigma}=\sigma^{\prime} \sqrt{\beta^{*} \widehat{\beta}}
$$

where the maximum beta function $\widehat{\beta}$ in the triplet is related to the collision beta through

$$
\widehat{\beta}=\frac{f^{2}}{\beta^{*}}
$$

The focal length $f$ in this equation is usually very close to a constant over a wide range of values of $\beta^{*}$, and so it is reasonable to re-write Equation 3 as

$$
\widehat{\sigma}=f \sigma^{\prime}
$$


It is necessary to fit at least $n$ maximum beam sigmas into the coil radius $r$ of the superconducting magnet

$$
n \widehat{\sigma} \leq r
$$

where $n$ is typically around 8 . Thus the limit to the angular beam size is

$$
\sigma^{\prime} \leq \frac{1}{n} \frac{r}{f}
$$

The focal length $f$ is significantly reduced (and the angular beam size $\sigma^{\prime}$ is significantly increased) when RHIC goes from a "dipole first" to a "quadrupole first" layout. New triplet quadrupoles also allow an additional increase in $\sigma^{\prime}$ - by further reducing $f$ by making the quadrupoles shorter, and by widening the bore radius $r$.

\subsection{Quadrupole parameters}

A good measure of the state-of-the-art is the maximum attainable field at the inner radius of the quadrupole coil

$$
B_{\text {coil }}=B^{\prime} r
$$

where $B^{\prime}$ is the quadrupole gradient. A good rule of thumb is that the maximum value of $B_{\text {coil }}$ is constant as $r$ is varied - the coil bore can only be increased at the expense of a reduction in quadrupole strength $B^{\prime}$. The maximum geometric strength of a quadrupole is more relevant to the optical scenarios presented below. It is given by

$$
K=\frac{B^{\prime}}{(B \rho)}
$$

where the maximum RHIC rigidity is

$$
(B \rho)=831.76[\mathrm{~T} / \mathrm{m}]
$$

for the top gold ion energy of $100 \mathrm{GeV} / \mathrm{u}$.

\begin{tabular}{|lcccc|}
\hline & & LHC IR & RHIC 99 & Quad first \\
\hline & & & & \\
\hline Coil radius, $r$ & {$[\mathrm{~mm}]$} & 35.0 & 65.0 & 65.0 \\
Maximum gradient, $B^{\prime}$ & {$[\mathrm{T} / \mathrm{m}]$} & 230 & 45.7 & 125 \\
Maximum coil field, $B_{\text {coil }}$ & {$[\mathrm{T}]$} & 8.05 & 2.97 & 8.11 \\
Geometric strength, $K$ & {$\left[\mathrm{~m}^{-2}\right]$} & 0.277 & 0.055 & 0.150 \\
& & & & \\
\hline
\end{tabular}

Table 1: Superconducting quadrupole parameters for RHIC and for LHC IR quads as built by FNAL and KEK.

Table 1 lists quadrupole parameters for the LHC IR quadrupoles built by FNAL and KEK [2], for RHIC as commissioned in 1999, and (under "Quad first") for the two scenarios discussed below. It is assumed that the new quads maintain the same $65 \mathrm{~mm}$ coil radius as the old quads, but with (almost) the same $8.11 \mathrm{~T}$ maximum coil field of the LHC quadrupoles. Maintaining the same coil bore is not an optimal choice, but it is a convenient simplification since the luminosity enhancement factor $H$

$$
H \equiv \frac{L_{\text {new }}}{L_{\text {old }}}=\left(\frac{\sigma_{\text {new }}^{\prime}}{\sigma_{\text {old }}^{\prime}}\right)^{2}=\frac{(r / f)_{\text {new }}^{2}}{(r / f)_{\text {old }}^{2}}
$$

becomes just

$$
H=\left(\frac{f_{\text {old }}}{f_{\text {new }}}\right)^{2}
$$

Table 1 shows that the maximum geometric strength of the new quadrupoles is $K=0.150 \mathrm{~m}^{-2}$. 


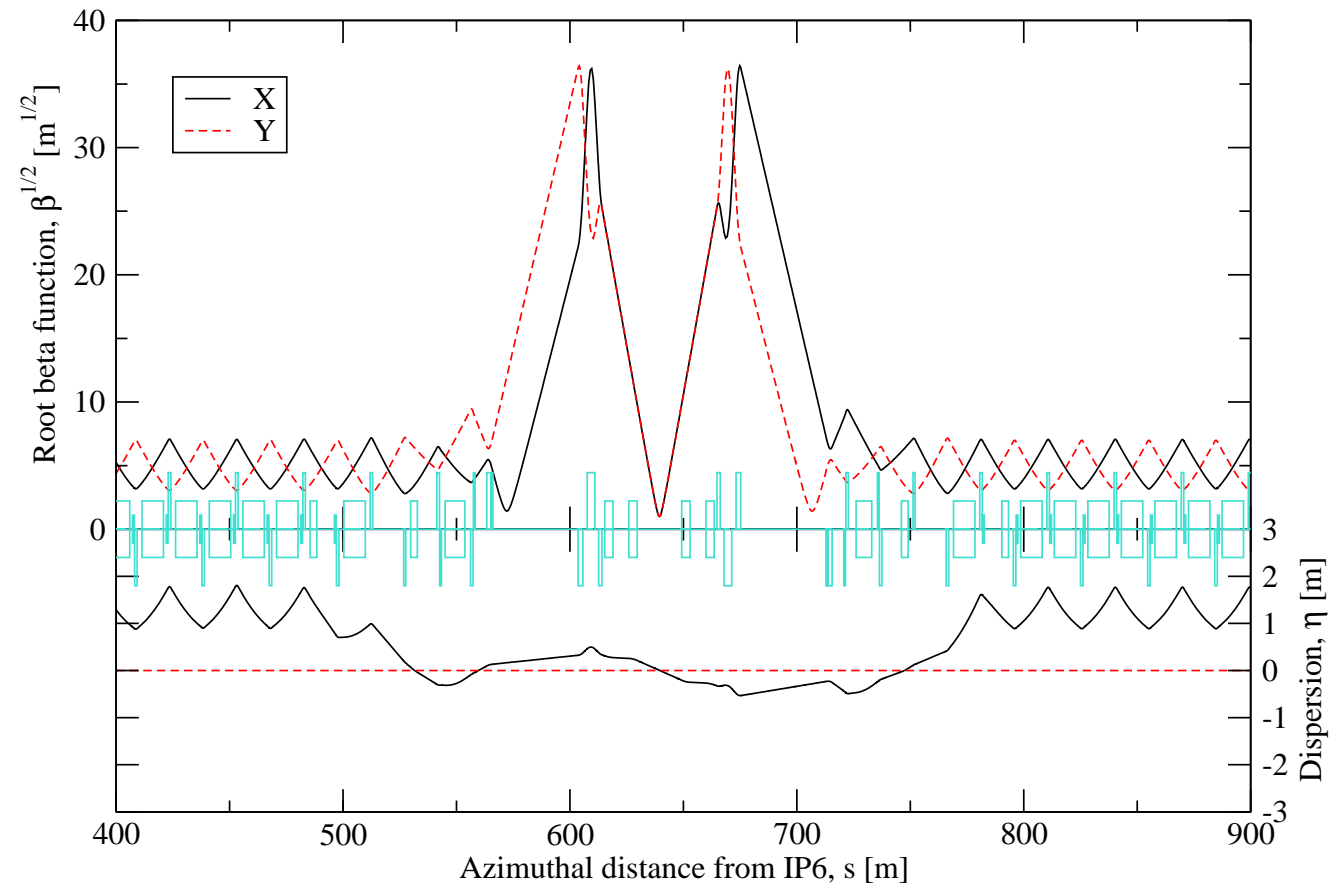

Figure 1: RHIC Interaction Region optics at storage, with $\beta^{*}=1 \mathrm{~m}$, in the configuration used from 1999 until the present. The inside face of the first beam separation dipole DX is at $\pm 9.8 \mathrm{~m}$ from the IP, followed by D0, and then by the triplet quadrupoles Q1, Q2, and Q3.

\section{Old and new scenarios}

Figure 1 shows the interaction region layout that has been used at all 6 RHIC IRs since collider commissioning in 1999. The first magnet (going outwards from the IP at $s=0$ ) is the first beam splitting dipole DX, with its inner face at a distance $s= \pm 9.8 \mathrm{~m}$ from the IP. In the storage optics shown here the collision beta is $\beta^{*}=1 \mathrm{~m}$ and the maximum beta is $\widehat{\beta}=1317 \mathrm{~m}$, for a focal length of $f=36.29 \mathrm{~m}$. These and other relevant parameters are listed in Table 2.

Figure 2 shows the first of two "quadrupole first" scenarios, with quadrupole Q1 just outside the $s= \pm 9.8 \mathrm{~m}$ boundary. This scenario is conservative, in that it is trivially compatible with the existing RHIC detectors. The dispersion function is fully matched, as also is the "survey" - arc magnets Q4 and Q5 et cetera are unmoved. The survey match is achieved by slightly increasing the spacing between the DX and D0 magnets. The (not fully optimized) solution shown here enables the collision beta to be reduced to $\beta^{*}=0.20 \mathrm{~m}$ while maintaining an approximately constant maximum beta function, so the focal length is reduced to $f=16.65 \mathrm{~m}$ for a luminosity enhancement factor of $H=4.75$.

Figure 3 shows the second scenario, with quadrupole Q1 inside the $s= \pm 9.8 \mathrm{~m}$ boundary (and with the inner face of Q2 at the boundary). This scenario is not quite compatible with STAR or PHENIX, but can easily be made compatible with a new detector like the Comprehensive Detector [1]. In the (not fully optimized) solution shown here the focal length is even further reduced, to $f=13.86 \mathrm{~m}$, for a luminosity enhancement factor of $H=6.86$.

\subsection{Coil radius and inter-quad spacing}

In both upgrade scenarios it has been assumed for simplicity that, in common with the current RHIC configuration

1. the triplet quadrupole coil radius remains $65 \mathrm{~mm}$

2. the inter-quad spacing is held constant

Both of these assumptions would be abandoned in a more thorough optimization of the problem. There is more luminosity to be gained by decreasing both the coil radius and the inter-quad spacing.

If the optimal coil radius is close to $35 \mathrm{~mm}$, then it would be possible to exploit the existing LHC quadrupole tooling at Fermilab and KEK. 


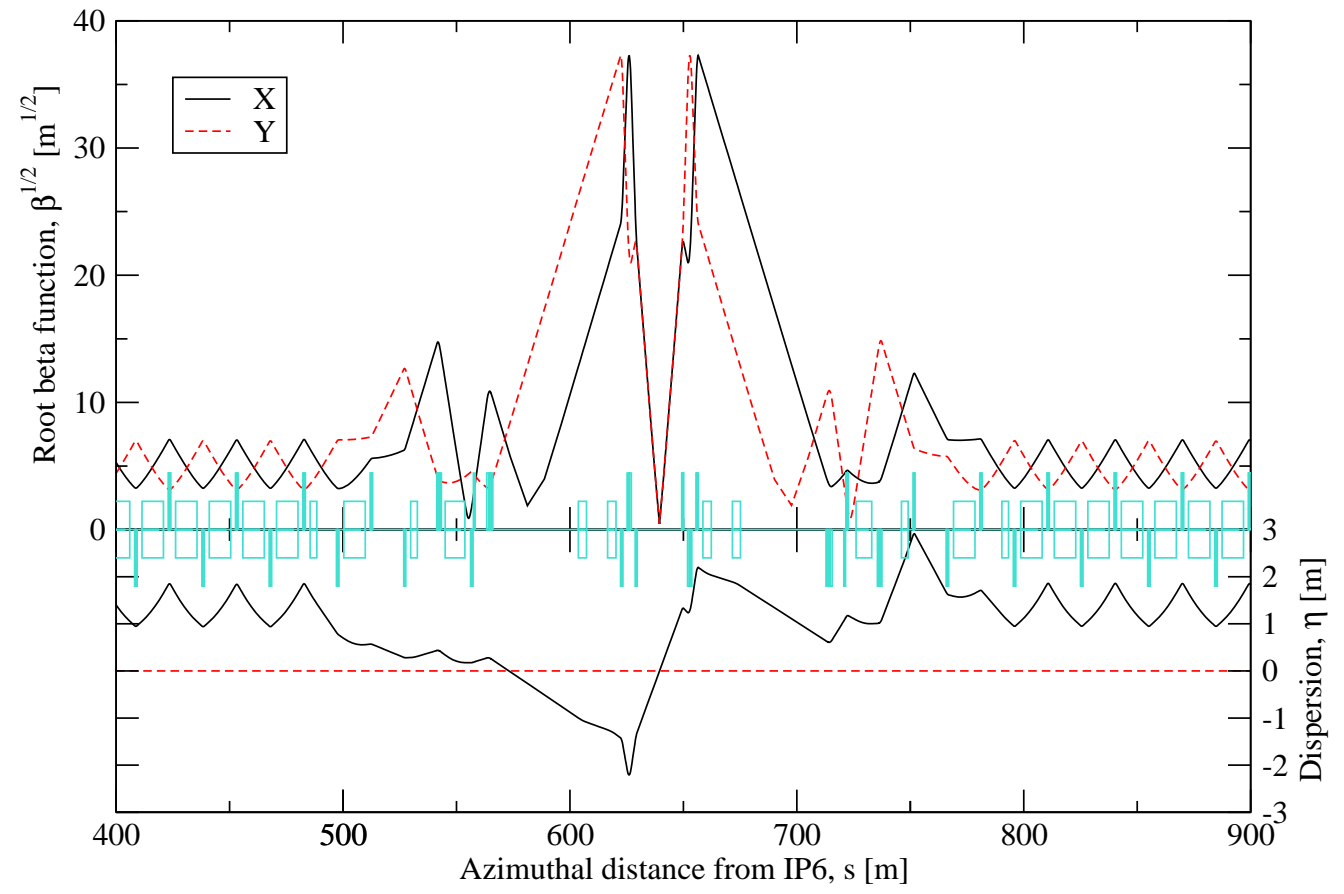

Figure 2: The outside 9.8 scenario, with the front face of the first quadrupole Q1 at $s= \pm 9.8$ meters from the IP. The collision beta is reduced to $\beta^{*}=0.20 \mathrm{~m}$ while the maximum beta remains almost constant, so that the focal length is reduced to $f=16.65 \mathrm{~m}$ and the luminosity is enhanced by a factor of 4.75 .

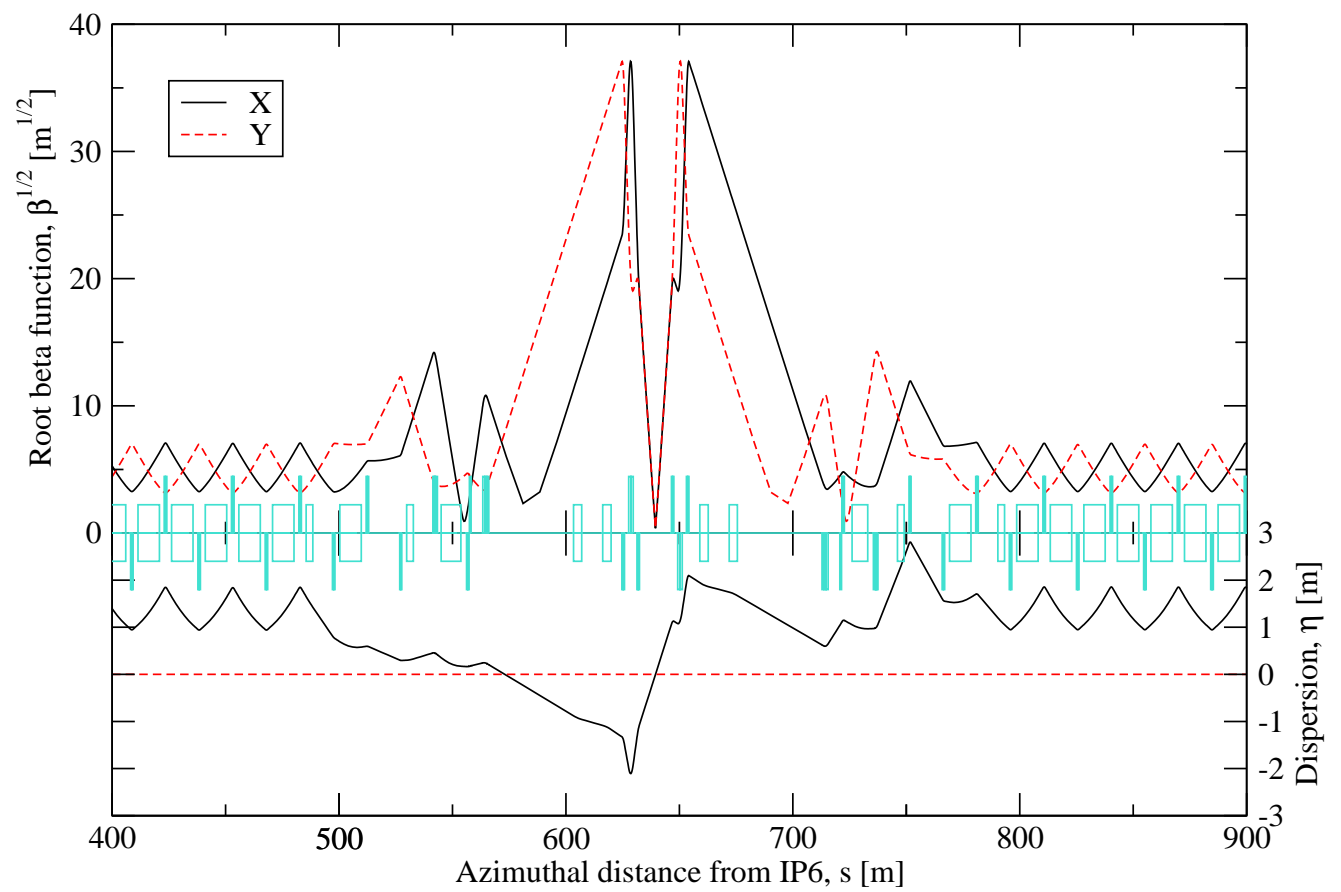

Figure 3: The inside 9.8 scenario, with the front face of the second quadrupole Q2 at $s= \pm 9.8$ meters from the IP, and with the first quadrupole inside. The collision beta is reduced even further, to $\beta^{*}=0.14 \mathrm{~m}$, while the maximum beta remains almost constant, so that the focal length is reduced to $f=13.86 \mathrm{~m}$ and the luminosity is enhanced by a factor of 6.86 . 


\begin{tabular}{|lcccc|}
\hline & & RHIC 99 & Outside 9.8 & Inside 9.8 \\
& & & & \\
\hline Magnetic length of Q1 & {$[\mathrm{m}]$} & 1.440 & 0.917 & 1.086 \\
Magnetic length of Q2 & {$[\mathrm{m}]$} & 3.392 & 1.845 & 1.987 \\
Magnetic length of Q3 & {$[\mathrm{m}]$} & 2.100 & 1.064 & 1.129 \\
Collision beta, $\beta^{*}$ & {$[\mathrm{~m}]$} & 1.0 & 0.20 & 0.14 \\
Maximum beta, $\widehat{\beta}$ & {$[\mathrm{m}]$} & 1317 & 1387 & 1373 \\
& & & & \\
Focal length, $f$ & {$[\mathrm{~m}]$} & 36.29 & 16.65 & 13.86 \\
Luminosity enhancement factor, $H$ & & 1 & 4.75 & 6.86 \\
& & & & \\
\hline
\end{tabular}

Table 2: Luminosity enhancement parameters for the "outside 9.8 " and "inside 9.8 " scenarios.

\section{Caveats and consequences}

Both beams go through common quadrupoles in the upgraded triplet, so both beams must have (nearly) identical rigidities. This is inconsistent with synchronized collisions when protons and gold ions counter-circulate. Further study is required to establish whether or not synchronized deuteron-gold collisions are possible.

1) Proton-gold collisions are not possible.

2) The viability of deuteron-gold collisions needs more study.

The bunch length must be smaller than (or about) the collision beta in order to avoid luminosity loss to the "hourglass" effect. Bunches as short as $0.1 \mathrm{~m}$ are not possible with the voltage gradients available with the current storage RF system (for nominal longitudinal emittances after Intra Beam Scattering). Somewhat shorter bunches also have other dynamical implications.

3) An enhanced RF system is necessary to make shorter bunches to reach the highest luminosities.

4) IBS rates, beampipe and BPM lead heating, and other short bunch effects need to be re-calculated.

Beam separation occurs further from the IP, and so the beta functions are significantly larger in the separation dipoles, while there are more long range beam-beam collisions.

5) Physical and good field apertures of the repositioned DX and D0 magnets need re-checking.

6) Long range (parasitic) beam-beam collisions need re-evaluation. Crossing angles may be advantageous.

7) The total path length change is negligible

If the luminosity is an order of magnitude higher, the burn rate becomes even more significant, unbalancing the current nominal operation scenario.

8) Optimum fill and store times and parameters need re-calculation.

There is no prima facie reason to expect that polarized proton performance will be adversely affected.

9) Re-calculate de-polarization resonance strengths up the ramp.

The natural chromaticity is most likely significantly increased (in magnitude) for an upgraded IR, potentially leading to deleterious effects. This is important in proportion to the number of IRs that are upgraded.

10) Include natural chromaticity in the optimization of the quad coil radius.

11) Evaluate second order chromaticity behavior.

12) Evaluate dynamics performance due to modified chromaticity correction sextupole strengths. 


\section{Summary}

Luminosity enhancement factors of at least 5 and 7 are possible with the first quadrupole outside or inside the "boundary" at 9.8 meters from the IP. An enhancement factor of 10 may be possible with further optimization of the optics - primarily by optimizing the superconducting coil radius, reducing the inter-quad spacing, and moving Q1 even closer to the IP.

If the optimum coil radius is close to the LHC value of $35 \mathrm{~mm}$, then it is possible to use the existing quadrupole tooling available at FNAL and KEK.

Luminosity enhancement by going to a quad first layout is in direct competition with the need for proton gold collisions. Many other relatively minor issues need further study. The only major issue is the need to determine whether deuteron gold collisions are, or are not, viable.

\section{Acknowledgments}

We are very grateful for discussions with Wolfram Fischer, Brett Parker, Peter Steinberg, and Dejan Trbojevic.

\section{References}

[1] RHIC II Physics and Perspectives for a New Comprehensive Detector, Workshop, Yale, April 2004, http://star.physics.yale.edu/users/bump/April2004RHICworkshop

[2] Low-beta triplets: towards the end of production, J. Strait, p. 166, Proceedings of the LHC Project Workshop Chamonix XIII, CERN-AB-2004-014 ADM, February 2004.

http://ab-div.web.cern.ch/ab-div/conferences/Chamonix/chamx2004/contents.html, 